\title{
Schrödinger's pure-state steering completed
}

\author{
F Herbut $\dagger$ \\ Serbian Academy of Sciences and Arts, Knez Mihajlova 35, 11000 Belgrade, Serbia
}

\begin{abstract}
Schrödinger investigated entanglement in two-particle state vectors by assuming measurement finding out if the nearby particle is in a given state vector $\psi_{1}$ or not. Without interaction with the distant particle, just on account of the entanglement, the distant particle is steered into a certain state vector. In Schrödinger's finite-dimensional case thus any distant-particle state vector can be reached. This theory was extended to infinite-dimensional spaces by the author. The present article completes the extension by throwing light on the fine structure of steering.
\end{abstract}

PACS numbers: 03.65.-w, 03.65.Ca, 03.65.Db, 03.65.Ud 


\section{Introduction}

When in 1935 Einstein et al. launched their revolutionary EPR paradox [1], many deep-thinking foundationally-minded physicists followed suit. Among them were Furry [2] and Schrödinger [3], 4]. The latter author introduced the now widely used concept of entanglement, but also that of disentanglement and of steering or distant steering [5]. Schrödinger's approach and indignation can be seen in his words [3] p. 556: "It is rather discomforting that the theory should allow a system to be steered or piloted into one or the other type of state at the experimenter's mercy in spite of his having no access to it." This is made even more clear in his next paper [4], p. 446: ".. in general a sophisticated experimenter can, by a suitable device which does not involve measuring non-commuting variables, produce a non-vanishing probability of driving the system into any state he chooses". He had two-particle pure states with non-singular reduced density operators, and finite dimensional state spaces of particles in mind. (This will be obvious after the detailed study in this article.)

Distant steering in case of two-particle state vectors that have reduced density operators with infinite-dimensional ranges were studied by the present author [6], [7]. The present article is actually a completion of the former study with insight in the fine structure of steering. Wiseman et al. extended steering to mixed two-particle states [8].

This study is focused on two-particle state vectors that have infinite-dimensional ranges of reduced density operators. (The theory is general, but the fine structure studied does not show up in the trivial, finite-dimensional case.)

\section{The distant state}

It is well known that one of the basic quantum-mechanical relations is the so-called trace rule, which expresses the probability $p(P, \rho)$ of occurrence of a quantum event (projector) $P$ in a quantum state (density operator) $\rho$ by the simple formula $p(P, \rho)=\operatorname{tr}(P \rho)$. 'Occurrence' is defined by measurement, but it is an astonishing quantum-mechanical fact (that one is usually not aware of) that this notion has a 'twodimensional multitude':

(i) One can take any observable (Hermitian operator) $A$ of which $P$ is an eigenprojector corresponding to an (arbitrary) eigenvalue $a$, i. e, an operator the spectral form of which is $A=a P+P^{\perp} A P^{\perp}$ where $P^{\perp} \equiv 1-P \quad$ (and the second term does not have the eigenvalue $a$ ). If in the measurement of $A$ the result $a$ is obtained, then one says that $P$ has occurred.

(ii) The observable specified in (i) can be measured in whatever way: ideally (the textbook case), when the Lüders formula [9] gives the change of state; in more general non-demolition measurement (older synonyms: repeatable measurement, or measurement of the first kind); in measurement in which the result is not preserved (non-repeatable or second-kind measurement). 
It is also not widely known that if one has a bipartite system in any correlated state (density operator) $\rho_{12}$, i. e., when $\rho_{12} \neq \rho_{1} \otimes \rho_{2}$, where the tensor factors are the reduced density operators, then, if a first-subsystem event $P_{1}$ occurs (in the sense defined in the preceding passages with the two multitudes of varieties), then the second subsystem ipso facto, i. e., without any interaction between the measuring instrument and the subsystem, makes transition from the subsystem state (reduced density operator) $\rho_{2} \equiv \operatorname{tr}_{1} \rho_{12}$ to the following state (density operator) in $\mathcal{H}_{2}$ :

$$
p^{-1} \operatorname{tr}_{1}\left(\rho_{12} P_{1}\right)
$$

where

$$
p \equiv \operatorname{tr}_{12}\left(P_{1} \rho_{12}\right)
$$

is the probability of the occurrence of $P_{1}$ in the state $\rho_{12}$. We write under the partial trace $P_{1}$ instead of $P_{1} \otimes I_{2}$, where $I_{2}$ is the identity operator in $\mathcal{H}_{2}$. (A proof of $(1 \mathrm{a}, \mathrm{b})$ is given in [10], subsection 6.B.)

We need two steps of confining ourselves to special cases from relation (1a) to come to Schrödinger's steering. We want to do this in the antilinear representation of bipartite state vectors (vectors of norm one) [7]. (The indices 1, 2, and 12 show in which space the entity is.)

\section{The antilinear representation}

There is an isomorphism from the tensor product $\mathcal{H}_{1} \otimes \mathcal{H}_{2}$, where the factors are complex separable (finite or countably infinite dimensional) Hilbert spaces (state spaces of the subsystems) to antilinear Hilbert-Schmidt operators $A_{a}$ that map $\mathcal{H}_{1}$ into $\mathcal{H}_{2}$, determined by partial scalar product:

$$
\begin{gathered}
\forall|\Phi\rangle_{12} \quad \rightarrow \quad A_{a}: \\
\forall|\psi\rangle_{1}: \quad\left(A_{a}|\psi\rangle_{1}\right)_{2} \equiv\left\langle\left.\psi\right|_{1} \mid \Phi\right\rangle_{12},
\end{gathered}
$$

where $\left\langle\left.\psi\right|_{1} \mid \Phi\right\rangle_{12}$ is the partial scalar product over subsystem 1.

Each antilinear operator $A_{a}$ defined by $(2 \mathrm{~b})$ determines its adjoint $A_{a}^{\dagger}$, which maps $\mathcal{H}_{2}$ into $\mathcal{H}_{1}$. The adjoint is determined via the relation

$$
\forall|\psi\rangle_{1},|\phi\rangle_{2}: \quad\left(A_{a}|\psi\rangle_{1},|\phi\rangle_{2}\right)_{2}=\left(|\psi\rangle_{1}, A_{a}^{\dagger}|\phi\rangle_{2}\right)_{1}^{*}
$$

where the brackets stand for scalar products, which are antilinear in the first factor, and the asterisk denotes complex conjugation.

Relation (2b) also implies $\operatorname{tr}\left(A_{a}^{\dagger} A_{a}\right)<\infty$. This is the relation that makes the antilinear operators $A_{a}$ and $A_{a}^{\dagger}$ Hilbert-Schmidt ones. 


\section{Two steps of special cases}

Now we make the first step of taking a special case of $(1 \mathrm{a}, \mathrm{b})$. By $P_{\chi_{i}}$ we denote the projector onto the one-dimensional subspace spanned by the unit vector $\chi_{i}$, and $i=1,2,12$ keeps track of the Hilbert space to which the entity belongs (even if it is superfluous, it is useful for transparency). Naturally, $P_{\chi_{i}} \psi_{i}=\chi_{i}\left(\chi_{i}, \psi_{i}\right)_{i}, \quad i=1,2,12$.

Theorem 1. If one has any bipartite state vector $\Phi_{12}$, i. e., $\rho_{12} \equiv P_{\Phi_{12}}$, and one goes over to the antilinear representation $A_{a}$ of $\Phi_{12}$, the occurrence of any first-subsystem event $P_{1}$ brings about the following second-subsystem state:

$$
p^{-1}\left[A_{a} P_{1} A_{a}^{\dagger}\right]_{2}
$$

where

$$
p \equiv\left(\Phi_{12}, P_{1} \Phi_{12}\right)_{12}
$$

is the probability of the event $P_{1}$ in the state $\Phi_{12}$.

Proof. As to the antilinear representation, we are going to utilize (2b) and (3), and the fact that numbers undergo complex conjugation when taken to the left from an antilinear operator. Let $\left\{\phi_{2}^{n}: \forall n\right\}$ be a complete orthonormal basis in $\mathcal{H}_{2}$, and let $\left\{\psi_{1}^{k}: \forall k\right\}$ be a complete orthonormal basis in $\overline{\mathcal{R}\left(P_{1}\right)}$, the topological closure of the range of $P_{1}: P_{1}=\sum_{k} P_{\psi_{1}^{k}}$. Then

$$
\begin{gathered}
\left(\phi_{2}^{n},\left[\operatorname{tr}_{1}\left(P_{\Phi_{12}} P_{1}\right)\right]_{2} \phi_{2}^{n^{\prime}}\right)_{2}=\sum_{k}\left(\psi_{1}^{k} \phi_{2}^{n}, P_{\Phi_{12}} \psi_{1}^{k} \phi_{2}^{n^{\prime}}\right)_{12}= \\
\sum_{k}\left(\psi_{1}^{k} \phi_{2}^{n}, \Phi_{12}\left(\Phi_{12}, \psi_{1}^{k} \phi_{2}^{n^{\prime}}\right)_{12}\right)_{12}=\sum_{k}\left(\psi_{1}^{k} \phi_{2}^{n^{\prime}}, \Phi_{12}\right)_{12}^{*}\left(\phi_{2}^{n}, A_{a} \psi_{1}^{k}\right)_{2}= \\
\sum_{k}\left(\phi_{2}^{n^{\prime}}, A_{a} \psi_{1}^{k}\right)_{2}^{*}\left(\phi_{2}^{n}, A_{a} \psi_{1}^{k}\right)_{2}=\sum_{k}\left(A_{a}^{\dagger} \phi_{2}^{n^{\prime}}, \psi_{1}^{k}\right)_{1}\left(\phi_{2}^{n}, A_{a} \psi_{1}^{k}\right)_{2}= \\
\sum_{k}\left(\psi_{1}^{k}, A_{a}^{\dagger} \phi_{2}^{n^{\prime}}\right)_{1}^{*}\left(\phi_{2}^{n}, A_{a} \psi_{1}^{k}\right)_{2}=\left(\phi_{2}^{n},\left[A_{a} \sum_{k} P_{\psi_{1}^{k}}\left[A_{a}^{\dagger} \phi_{2}^{n^{\prime}}\right]_{1}\right]_{2}\right)_{2}= \\
\left(\phi_{2}^{n},\left[A_{a} P_{1}\left[A_{a}^{\dagger} \phi_{2}^{n^{\prime}}\right]_{1}\right]_{2}\right)_{2}=\left(\phi_{2}^{n},\left[A_{a} P_{1} A_{a}^{\dagger}\right]_{2} \phi_{2}^{n^{\prime}}\right)_{2} \cdot
\end{gathered}
$$

The second step of taking a special case of $(4 a, b)$ is confining ourselves to elementary first-subsystem events (ray projectors) $P_{1} \equiv P_{\psi_{1}}$, where $\psi_{1}$ is an arbitrary state vector.

Theorem 2. If an elementary event $P_{\psi_{1}}$ occurs on the first subsystem in a state vector $\Phi_{12}$ (or $A_{a}$ ), then the second subsystem finds itself in the state described by the state vector

$$
A_{a} \psi_{1} /\left\|A_{a} \psi_{1}\right\|
$$


and the probability of the occurrence of $P_{\psi_{1}}$ is

$$
p=\left\|A_{a} \psi_{1}\right\|^{2}
$$

Proof. As to the claimed probability (5b), from (4b) one has

$$
p=\operatorname{tr}_{12}\left(P_{\Phi_{12}} P_{\psi_{1}}\right) .
$$

Introducing complete orthonormal bases $\left\{\psi_{1}^{k}: \forall k ; \psi_{1}^{k=1} \equiv \psi_{1}\right\}$ and $\left\{\phi_{2}^{n}: \forall n\right\}$, one obtains

$$
p=\sum_{n}\left(\psi_{1} \phi_{2}^{n}, P_{\Phi_{12}} \psi_{1} \phi_{2}^{n}\right)_{12}
$$

Applying the projector, and taking out one scalar product from the other, one further has

$$
\begin{gathered}
p=\sum_{n}\left(\Phi_{12}, \psi_{1} \phi_{2}^{n}\right)_{12}\left(\psi_{1} \phi_{2}^{n}, \Phi_{12}\right)_{12}= \\
\sum_{n}\left(\psi_{1} \phi_{2}^{n}, \Phi_{12}\right)_{12}^{*}\left(\phi_{2}^{n}, A_{a} \psi_{1}\right)_{2}=\sum_{n}\left(\phi_{2}^{n}, A_{a} \psi_{1}\right)_{2}^{*}\left(\phi_{2}^{n}, A_{a} \psi_{1}\right)_{2}=\left\|A_{a} \psi_{1}\right\|^{2} .
\end{gathered}
$$

To derive claim (5a), we start with (4a), and we utilize the above basis in $\mathcal{H}_{2}$.

$$
\begin{gathered}
\left(\phi_{2}^{n}, p^{-1}\left[A_{a} P_{\psi_{1}} A_{a}^{\dagger}\right]_{2} \phi_{2}^{n^{\prime}}\right)_{2}=\left(\phi_{2}^{n},\left[p^{-1 / 2} A_{a} \psi_{1}\right]_{2}\left(\psi_{1},\left[p^{-1 / 2} A_{a}^{\dagger} \phi_{2}^{n^{\prime}}\right]_{1}\right)_{1}\right)_{2}= \\
\left(\psi_{1},\left[p^{-1 / 2} A_{a}^{\dagger} \phi_{2}^{n^{\prime}}\right]_{1}\right)_{1}^{*}\left(\phi_{2}^{n},\left[p^{-1 / 2} A_{a} \psi_{1}\right]_{2}\right)_{2}=\left(\left[p^{-1 / 2} A_{a} \psi_{1}\right]_{2}, \phi_{2}^{n^{\prime}}\right)_{2}\left(\phi_{2}^{n},\left[p^{-1 / 2} A_{a} \psi_{1}\right]_{2}\right)_{2}= \\
\left(\phi_{2}^{n},\left[p^{-1 / 2} A_{a} \psi_{1}\right]_{2}\left(\left[p^{-1 / 2} A_{a} \psi_{1}\right]_{2}, \phi_{2}^{n^{\prime}}\right)_{2}\right)=\left(\phi_{2}^{n}, P_{\left[p^{-1 / 2} A_{a} \psi_{1}\right]_{2}} \phi_{2}^{n^{\prime}}\right)_{2} \cdot
\end{gathered}
$$

One should note that after the second equality the scalar product is complex conjugated because before the first equality, $A_{a}$ is seen to act after the projector, hence also on the numbers that come out as a result of the projection. Contrariwise, if we read the next to last expression one step backwards, the scalar product is extracted without complex conjugation though it is to the right of $A_{a}$. The reason is that $A_{a}$ acts in $\mathcal{H}_{1}$, and the scalar product (a number) appears in $\mathcal{H}_{2}$ after the action of $A_{a}$.

Schrödinger's steering is defined for the occurrence of an arbitrary elementary firstsubsystem event $P_{\psi_{1}}$ in an arbitrary bipartite state vector $\Phi_{12}$. As it is shown in Theorem 2, this boils down to mapping $\mathcal{H}_{1}$ into $\mathcal{H}_{2}$ by $A_{a}$ (the antilinear representative of $\left.\Phi_{12}\right)$.

\section{Polar factorization}

To bring out the full power of the antilinear representation, one should perform the two polar factorizations of $A_{a}$ [11]:

$$
A_{a}=U_{a} \rho_{1}^{1 / 2}
$$




$$
A_{a}=\rho_{2}^{1 / 2} U_{a} Q_{1}
$$

where $\rho_{i} \equiv \operatorname{tr}_{j} P_{\Phi_{12}}, \quad i, j=1,2, \quad i \neq j$ is the $i$-th subsystem state (reduced density operator), and $U_{a}$ is an antilinear unitary operator mapping the topologically closed range $\overline{\mathcal{R}\left(\rho_{1}\right)}$ onto the topologically closed range $\overline{\mathcal{R}\left(\rho_{2}\right)}$ (these subspaces are always equally dimensional), and, finally, $Q_{1}$ is the range-projector of $\rho_{1}$.

The operator $U_{a}$ is called the correlation operator. It is the only precise mathematical entity expressing the quantum correlations inherent in a bipartite state (known to the author).

Remark 1. As it is seen in (6b), Schrödinger's steering maps $\mathcal{H}_{1}$ into $\mathcal{R}\left(\rho_{2}^{1 / 2}\right)$. Actually, it is a surjection, i. e., an "onto" map [6]. This is, of course, non-trivial only in case of infinite-dimensional ranges (of $\rho_{i}, i=1,2$ ), when one should have in mind the known proper inclusion relations:

$$
\mathcal{R}(\rho) \subset \mathcal{R}\left(\rho^{1 / 2}\right) \subset \overline{\mathcal{R}(\rho)}
$$

valid for any density operator with infinite-dimensional range.

\section{Largest probability of steering}

We proceed by analyzing (5a,b) to gain detailed insight in Schrödinger's steering.

Theorem 3. A) Two first-subsystem state vectors $\psi_{1}$ and $\psi_{1}^{\prime}$ give, upon measurement, the same steering in subsystem 2 if and only if

(i) the range-projections are positively collinear:

$$
Q_{1} \psi_{1}=c Q_{1} \psi_{1}^{\prime}, \quad c>0
$$

or equivalently

(ii) if they determine by projection the same state vector in $\overline{\mathcal{R}\left(\rho_{1}\right)}$ :

$$
Q_{1} \psi_{1} /\left\|Q_{1} \psi_{1}\right\|=Q_{1} \psi_{1}^{\prime} /\left\|Q_{1} \psi_{1}^{\prime}\right\| .
$$

B) Of all elementary events $P_{\psi_{1}}$ in $\mathcal{H}_{1}$ that give one and the same state vector in $\mathcal{H}_{2}$ by steering largest probability of occurrence has the one that lies entirely in $\overline{\mathcal{R}\left(\rho_{1}\right)}$, or, equivalently, the component of which in the null space of $A_{a}$ is zero.

Proof. A) Sufficiency. Let (8a) be valid. Since $\rho_{1}=\rho_{1} Q_{1}$, and $\rho_{1}^{1 / 2}=\rho_{1}^{1 / 2} Q_{1}$, (6a) implies $A_{a}=A_{a} Q_{1}$. Hence, applying $A_{a}$ to (8a), one obtains $A_{a} \psi_{1}=c A_{a} \psi_{1}^{\prime}$, and $\left\|A_{a} \psi_{1}\right\|=c\left\|A_{a} \psi_{1}^{\prime}\right\|$. Finally,

$$
A_{a} \psi_{1} /\left\|A_{a} \psi_{1}\right\|=A_{a} \psi_{1}^{\prime} /\left\|A_{a} \psi_{1}^{\prime}\right\|
$$

Necessity. If relation (9) is valid, then

$$
A_{a}\left(\psi_{1} /\left\|A_{a} \psi_{1}\right\|-\psi_{1}^{\prime} /\left\|A_{a} \psi_{1}^{\prime}\right\|\right)=0=Q_{1}\left(\psi_{1} /\left\|A_{a} \psi_{1}\right\|-\psi_{1}^{\prime} /\left\|A_{a} \psi_{1}^{\prime}\right\|\right)
$$


( $A_{a}$ and $Q_{1}$ have the same null space). Finally,

$$
Q_{1} \psi_{1}=\left(\left\|A_{a} \psi_{1}\right\| /\left\|A_{a} \psi_{1}^{\prime}\right\|\right) Q_{1} \psi_{1}^{\prime}
$$

Thus, (8a) is satisfied.

Clearly, (8b) implies (8a). Conversely, (8a) gives $\left\|Q_{1} \psi_{1}\right\|=c\left\|Q_{1} \psi_{1}^{\prime}\right\|$. Relation (8b) ensues from (8a) and this relation.

B) Relation (5b) implies

$$
p=\left\|A_{a} \psi_{1}\right\|^{2}=\left\|A_{a} Q_{1} \psi_{1}\right\|^{2}=\left(\left\|Q_{1} \psi_{1}\right\|^{2}\right)\left[\left\|A_{a}\left(Q_{1} \psi_{1} /\left\|Q_{1} \psi_{1}\right\|\right)\right\|^{2}\right] .
$$

All vectors specified in (8b) have the second factor after the last equality in (10) in common. Therefore, the probability is largest when the first factor (after the last equality in $(10))$ is largest, i. e., when it is one.

\section{The fine structure of infinite-dimensional ranges}

In this section we make a deviation from our two-particle study to one Hilbert space and a given density operator with an infinite-dimensional range in it.

Remark 2. Let $\rho$ be a density operator with an infinite-dimensional range. Writing " $\oplus$ " for the union of disjoint sets, and " $\ominus$ " when set-theoretically subtracting a subset from a larger set, the proper-inclusion chain (7) implies

$$
\overline{\mathcal{R}(\rho)}=\mathcal{R}(\rho) \oplus\left(\mathcal{R}\left(\rho^{1 / 2}\right) \ominus \mathcal{R}(\rho)\right) \oplus\left(\overline{\mathcal{R}(\rho)} \ominus \mathcal{R}\left(\rho^{1 / 2}\right)\right)
$$

Lemma 1. Let $\left\{\psi_{k}: \forall k\right\}$ be a complete orthonormal eigenbasis of $\rho$ in $\overline{\mathcal{R}(\rho)}$, and let $\left\{r_{k}: \forall k\right\}$ be the corresponding positive spectrum of $\rho$ (with possible repetitions of equal eigenvalues in general). Let, further, $\overline{\mathcal{R}(\rho)} \ni \psi=\sum_{k} a_{k} \psi_{k}$, with $\forall k: a_{k} \in \mathbf{C}$ be an arbitrary element, i. e., $\quad \sum_{k}\left|a_{k}\right|^{2}<\infty$. Then

$$
\psi \in \mathcal{R}(\rho) \quad \Leftrightarrow \quad \sum_{k}\left|r_{k}^{-1} a_{k}\right|^{2}<\infty
$$

and

$$
\psi \in \mathcal{R}\left(\rho^{1 / 2}\right) \quad \Leftrightarrow \quad \sum_{k}\left|r_{k}^{-1 / 2} a_{k}\right|^{2}<\infty .
$$

Proof. $\{\Leftarrow$ in (12)\}. Assuming the validity of the second expression in (12), we define $\phi \equiv \sum_{k} r_{k}^{-1} a_{k} \psi_{k}$. Then one has $\psi=\rho \phi$, i. e., the first expression in (12) holds true.

$\{\Rightarrow$ in (12) $\}$. If $\psi$ belongs to the range, there exists $\phi=\sum_{k} b_{k} \psi_{k}, \sum_{k}\left|b_{k}\right|^{2}<$ $\infty$, and $\rho \phi=\psi$. Since $\forall k: a_{k}=r_{k} b_{k}$, one has $\forall k: \sum_{k}\left|r_{k}^{-1} a_{k}\right|^{2}<\infty$.

Equivalence (13) is proved analogously. 
Lemma 2. The square root $\rho^{1 / 2}$ of any density operator $\rho$ with an infinitedimensional range maps in an one-to-one way $\overline{\mathcal{R}(\rho)}$ onto $\mathcal{R}\left(\rho^{1 / 2}\right)$, and by this it maps $\mathcal{R}\left(\rho^{1 / 2}\right)$ onto $\mathcal{R}(\rho)$, and $\left\{\overline{\mathcal{R}(\rho)} \ominus \mathcal{R}\left(\rho^{1 / 2}\right)\right\}$ onto $\left\{\mathcal{R}\left(\rho^{1 / 2}\right) \ominus \mathcal{R}(\rho)\right\}$, i. e., $(14 \mathrm{a}-\mathrm{c})$ is valid:

$$
\begin{gathered}
\mathcal{R}\left(\rho^{1 / 2}\right) \oplus\left\{\overline{\mathcal{R}(\rho)} \ominus \mathcal{R}\left(\rho^{1 / 2}\right)\right\}=\overline{\mathcal{R}(\rho)} \\
\downarrow \\
\downarrow \\
\mathcal{R}(\rho) \oplus\left\{\mathcal{R}\left(\rho^{1 / 2}\right) \ominus \mathcal{R}(\rho)\right\}=\mathcal{R}\left(\rho^{1 / 2}\right) .
\end{gathered}
$$

Proof. That $\rho^{1 / 2}$ maps $\overline{\mathcal{R}(\rho)}$ into $\mathcal{R}\left(\rho^{1 / 2}\right)$ is obvious from (6b). To prove that it is an "onto" map, let $\psi=\sum_{k} a_{k} \psi_{1}$ (cf Lemma 1) be an arbitrary element of the latter range. Then, according to (13), also $\phi \equiv \sum_{k} r_{k}^{-1 / 2} a_{k} \psi_{k}$ is an element of $\overline{\mathcal{R}(\rho)}$. Applying $\rho^{1 / 2}$ to it, we obtain $\psi$. Assuming ab contrario that $\phi, \phi^{\prime} \in \overline{\mathcal{R}(\rho)}$, $\phi \neq \phi^{\prime}$, and $\rho^{1 / 2} \phi=\rho^{1 / 2} \phi^{\prime}$, one arrives at $\rho^{1 / 2}\left(\phi-\phi^{\prime}\right)=0$, i.e., a non-zero element is taken into zero. This is not possible because $\rho^{1 / 2}$ has the same null space as $\rho$, and it is the orthocomplement of $\overline{\mathcal{R}(\rho)}$.

The first arrow in (14b), i. e., the map $\rho^{1 / 2}$ that it denotes, is obvious in the "into" sense because $\rho^{1 / 2} \rho^{1 / 2}=\rho$. Let $\psi=\sum_{k} a_{k} \psi_{k}$ be an arbitrary element of $\mathcal{R}(\rho)$. Then, according to (12), $\sum_{k}\left|r_{k}^{-1} a_{k}\right|^{2}<\infty$. Then also $\sum_{k}\left|r_{k}^{-1 / 2} a_{k}\right|^{2}<\infty \quad$ (compare the first inclusion in (7) with (12) and (13)). Hence, we can define $\phi \equiv \sum_{k} r_{k}^{-1 / 2} a_{k}$, and we have $\rho^{1 / 2} \phi=\psi$. Thus, we are dealing with an "onto" map.

Finally, the last claim is an immediate consequence of the preceding two, as easily seen.

\section{Back to steering}

It was shown in previous work [1] that the correlation operator not just maps $\overline{\mathcal{R}\left(\rho_{1}\right)}$ onto $\overline{\mathcal{R}\left(\rho_{2}\right)}$. It takes by similarity transformation the positive part of one reduced density operator into that of the other:

$$
\rho_{2}=U_{a} \rho_{1} U_{a}^{-1} Q_{2}
$$

where $Q_{2}$ is the range projector of $\rho_{2}$.

Lemma 3. The correlation operator preserves decomposition (11):

$$
\begin{aligned}
U_{a} \overline{\mathcal{R}\left(\rho_{1}\right)} & =\overline{\mathcal{R}\left(\rho_{2}\right)}, \\
U_{a} \mathcal{R}\left(\rho_{1}\right) & =\mathcal{R}\left(\rho_{2}\right), \\
U_{a}\left(\mathcal{R}\left(\rho_{1}^{1 / 2}\right) \ominus \mathcal{R}\left(\rho_{1}\right)\right) & =\left(\mathcal{R}\left(\rho_{2}^{1 / 2}\right) \ominus \mathcal{R}\left(\rho_{2}\right)\right),
\end{aligned}
$$




$$
U_{a}\left(\overline{\mathcal{R}\left(\rho_{1}\right)} \ominus \mathcal{R}\left(\rho_{1}^{1 / 2}\right)\right)=\left(\overline{\mathcal{R}\left(\rho_{2}\right)} \ominus \mathcal{R}\left(\rho_{2}^{1 / 2}\right)\right)
$$

Proof. In Lemma 1 we made the choice $\forall k: \rho_{1} \psi_{1}^{k}=r_{k} \psi_{1}^{k}$. Applying the correlation operator, one obtains $\forall k:\left(U_{a} \rho_{1} U_{a}^{-1}\right)\left(U_{a} \psi_{1}^{k}\right)=r_{k}\left(U_{a} \psi_{1}^{k}\right)$. Defining $\forall k: \psi_{2}^{k} \equiv U_{a} \psi_{1}^{k}$, one can, account of (15), write $\forall k: \rho_{2} \psi_{2}^{k}=r_{k} \psi_{2}^{k}$. Since $\mathcal{R}\left(\rho_{i}\right) i=1,2$ is the linear manifold spanned by the eigenvectors $\left\{\psi_{i}^{k}: \forall k\right\}, i=1,2$, (16b) is valid.

According to (13), $\quad \sum_{k}\left|r_{k}^{-1 / 2} a_{k}\right|^{2}<\infty$ is satisfied for every element $\psi_{1}=$ $\sum_{k} a_{k} \psi_{1}^{k}$ that belongs to $\mathcal{R}\left(\rho_{1}^{1 / 2}\right)$. Applying $U_{a}$, one has $\left(U_{a} \psi_{1}\right)=\sum_{k} a_{k}^{*} \psi_{2}^{k}$, and $\sum_{k}\left|r_{k}^{-1 / 2} a_{k}^{*}\right|^{2}=\sum_{k}\left|r_{k}^{-1 / 2} a_{k}\right|^{2}<\infty$. Thus, $U_{a} \mathcal{R}\left(\rho_{1}^{1 / 2}\right)=\mathcal{R}\left(\rho_{2}^{1 / 2}\right)$. The rest in the claim is evident.

Theorem 4. The antilinear representative $A_{a}$ of a given bipartite state vector $\Phi_{12}$ (cf $\left.(2 \mathrm{a}, \mathrm{b})\right)$ that implies reduced density operators with infinite-dimensional ranges maps in an one-to-one way $\overline{\mathcal{R}\left(\rho_{1}\right)}$ onto $\mathcal{R}\left(\rho_{2}^{1 / 2}\right)$, and by this it maps $\mathcal{R}\left(\rho_{1}^{1 / 2}\right)$ onto $\mathcal{R}\left(\rho_{2}\right)$, and $\left\{\overline{\mathcal{R}\left(\rho_{1}\right)} \ominus \mathcal{R}\left(\rho_{1}^{1 / 2}\right)\right\}$ onto $\left\{\mathcal{R}\left(\rho_{2}^{1 / 2}\right) \ominus \mathcal{R}\left(\rho_{2}\right)\right\}$. This is made more transparent by the following relations:

$$
\begin{gathered}
\mathcal{R}\left(\rho_{1}^{1 / 2}\right) \oplus\left\{\overline{\mathcal{R}\left(\rho_{1}\right)} \ominus \mathcal{R}\left(\rho_{1}^{1 / 2}\right)\right\}=\overline{\mathcal{R}\left(\rho_{1}\right)} \\
\downarrow \\
\downarrow \\
\mathcal{R}\left(\rho_{2}\right) \oplus\left\{\mathcal{R}\left(\rho_{2}^{1 / 2}\right) \ominus \mathcal{R}\left(\rho_{2}\right)\right\}=\mathcal{R}\left(\rho_{2}^{1 / 2}\right) .
\end{gathered}
$$

Proof. The claim of the theorem is evident having in mind the polar factorization (6b) of $A_{a}$, Lemma 3 and Lemma 2 in application to $\rho_{2}$.

\section{Conclusion}

In the formalism t he map $A_{a}$ that represents antilinearly any given bipartite state vector $\Phi_{12}$ performs actually the Schrödinger steering. If the composite-system state vector implies infinite-dimensional reduced density operators $\rho_{i}, i=1,2$, then the mapping has a fine structure:

(i) If the measurement of $\psi_{1}$ is that of an eigenvector of $\rho_{1}$ corresponding to a positive eigenvalue, then actually the corresponding eigenvector $\psi_{2}=U_{a} \psi_{1}$ is distantly measured. This simplest case was extensively studied in [11] and [12] in the non-selective version of measurement, when all results are taken into account in contrast to Schrödinger's steering, in which the selective version of measurement is considered with only one result - that of obtaining 1 for $P_{\psi_{1}}$. 
(ii) All other elements of $\mathcal{R}\left(\rho_{2}\right)$ can be obtained by steering that results from direct measurement of a vector $\psi_{1}$ from $\mathcal{R}\left(\rho_{1}^{1 / 2}\right)$. This case was studied in detail, again in the non-selective version of measurement, in [7]. Finally:

(iii) The elements of $\left(\mathcal{R}\left(\rho_{2}^{1 / 2}\right) \ominus \mathcal{R}\left(\rho_{2}\right)\right)$ can be reached by steering when direct measurement of vectors from $\left(\overline{\mathcal{R}\left(\rho_{1}\right)} \ominus \mathcal{R}\left(\rho_{1}^{1 / 2}\right)\right)$ is performed.

Besides, the vectors from the range of $\rho_{1}$ give, by selective measurement, the largest probability. Hence, the null space of $\rho_{1}$ is best discarded in steering.

The paradoxical physical meaning of distant steering is not discussed in this article. Quantum-mechanical insight in the nice EPR-type entanglement experiments of Scully et al. [13], [14] (a thought and a real experiment) gained by the present author recently [15], 16] has led to the conclusion that distant correlations are paradoxical only in the Einsteinian absolute-property interpretation of quantum mechanics. If one takes resort to the alternative, the relative-property interpretation, a kind of Everettian approach, then nothing is paradoxical.

[1] A. Einstein, B. Podolsky, and N. Rosen, Phys. Rev. 47, 777 (1935).

[2] W. H. Furry, Phys. Rev. 49, 393 (1936).

[3] E. Schrödinger, Proc. Cambridge Phil. Soc. 31, 555 (1935).

[4] E. Schrödinger, Proc. Cambridge Phil. Soc. 32, 446 (1936).

[5] R. W. Spekkens, Phys. Rev A 75, 032110 (2007); also available as quant-ph/0401052.

[6] M. Vujičić and F. Herbut, J. Phys A: Math. Gen. 21, 2931 (1988).

[7] F. Herbut, J. Math. Phys. 47, 122103 (2006). Reprinted in Virtual J. Quant. Inf., Dec. 2006 issue; also available as quant-ph/0609073

[8] H. M. Wiseman, S. J. Jones, and A. C. Doherty, Phys. Rev. Lett. 98, 140402 (2007); also available as quant-ph/0612147.

[9] G. Lüders, Ann. der Physik (in German) 8, 322 (1951). A. Messiah Quantum mechanics vol. I, (North Holland, Amsterdam, 1961), p 333. C. Cohen-Tannoudji, B. Diu, and F. Laloe, Quantum Mechanics vol. I (Wiley-Interscience, New York, 1977), p. 221 (Fifth Postulate).

[10] F. Herbut, Ann. Phys. (N. Y.) 55, 271 (1969).

[11] F. Herbut, M. Vujičić, Ann. Phys (N. Y.) 96, 382 (1976).

[12] M. Vujičić and F. Herbut, J. Math. Phys. 25, 2253 (1984).

[13] M. O. Scully, B.-G. Englert and H. Walther, Nature 351, 111 (1991).

[14] Y.-H. Kim, R. Yu, S. P. Kulik, Y. Shih and M.O. Scully, Phys. Rev. Lett. 84, 1 (2000); also quant-ph/9903047.

[15] F. Herbut, On the EPR-type entanglement in the experiments of Scully et al. I. The micromaser case and delayed-choice quantum erasure. quant-ph/0808.3176.

[16] F. Herbut, On EPR-type entanglement in the experiments of Scully et al. II. Insight in the real random delayed-choice erasure experiment. quant-ph/0808.3177. 